\title{
Hallucinations, delusions, and cognitive decline in Alzheimer's disease
}

\author{
Robert S Wilson, David W Gilley, David A Bennett, Laurel A Beckett, Denis A Evans
}

\begin{abstract}
Objectives-To examine the occurrence of hallucinations and delusions in Alzheimer's disease over a 4 year period and their association with rate of cognitive decline.

Methods-A cohort of 410 persons with clinically diagnosed Alzheimer's disease underwent annual clinical evaluations over a 4 year period. Participation in follow up exceeded $90 \%$ in survivors. Evaluations included structured informant interview, from which the presence or absence of hallucinations and delusions was ascertained, and detailed testing of cognitive function. The primary cognitive outcome measure was a composite cognitive score based on 17 individual performance tests. The mini mental state examination (MMSE) and summary measures of memory, visuoconstruction, repetition, and naming were used in secondary analyses.
\end{abstract}

Results-At baseline, hallucinations (present in $41 \%$ ) and delusions (present in $55 \%)$ were common and associated with lower cognitive function. In analyses that controlled for baseline level of cognitive function, demographic variables, parkinsonism, and use of antipsychotic medications, hallucinations, but not delusions, were associated with more rapid cognitive decline on each cognitive measure. In the primary model, there was a $47 \%$ increase in the average annual rate of decline on a composite cognitive measure in those with baseline hallucinations compared with those without them. This effect was mainly due to a subgroup with both auditory and visual hallucinations.

Conclusion-These findings suggest that the presence of hallucinations is selectively associated with more rapid cognitive decline in Alzheimer's disease. (F Neurol Neurosurg Psychiatry 2000;69:172-177)

Keywords: Alzheimer's disease; hallucinations; cognitive decline

Department of

Psychology, Rush

University and

Rush-Presbyterian-St

Luke's Medical Center,

Chicago, IL, USA

R S Wilson

D W Gilley

Correspondence to: Dr Robert S Wilson

Received 1 November 1999 and in final form

31 March 2000

Accepted 14 April 2000

Persons with Alzheimer's disease often show signs of hallucinations or delusional thinking at some point in the disease course. ${ }^{1}$ It has been difficult, however, to establish the relation of these psychotic features to the progressive decline in cognitive function which characterises the disease. Several longitudinal studies have found the presence of psychotic signs in Alzheimer's disease to be associated with more rapid cognitive decline, ${ }^{2-7}$ but evidence of this association has been inconsistent ${ }^{8-11}$ or lacking ${ }^{12-16}$ in other studies.

These variable results likely reflect several factors. Firstly, the presence or absence of psychosis has been determined from a single evaluation in some studies ${ }^{25-1416}$ but from multiple evaluations in others. ${ }^{34615}$ Secondly, hallucinations and delusions, the main signs of psychosis in persons with Alzheimer's disease, have been analysed separately in some studies $^{2} 891214-16$ but together in others. ${ }^{3-7} 101113$ Thirdly, in most studies of this issue, assessment of cognitive function has been restricted to the mini mental state examination (MMSE). ${ }^{17}$ However, because of its brevity, the MMSE has limitations in assessing change (for example, floor and ceiling effects, ${ }^{18}$ practice effects ${ }^{19}$ ), and because of its global nature, the MMSE may not identify effects confined to specific cognitive domains. Fourthly, some studies have not adequately controlled for demographic (for example, age, education) or clinical (for example, antipsychotic medications, parkinsonism) variables which may influence the association of psychotic signs with cognitive decline. Fifthly, the ability to model change in cognitive function has been limited in some studies by factors such as low follow up participation, number and spacing of observations, and duration of the study period.

In the present study, we examined the occurrence of hallucinations and delusions over a 4 year period in persons with Alzheimer's disease and the relation of these behaviours to patterns of cognitive decline. A cohort of more than 400 patients was evaluated annually, with over $90 \%$ participation in follow up among survivors. Evaluations included structured interview of a knowledgeable informant about the occurrence of hallucinations and delusions and administration of a battery of cognitive performance tests. Analyses assessed whether the presence of hallucinations or delusions at baseline, or over the full study period, was associated with cognitive decline, and whether results were influenced by selected demographic or clinical variables or by how cognitive function was measured.
Methods

SUBJECTS

This cohort has been described elsewhere. ${ }^{20}$ Eligibility required a clinical diagnosis of Alzheimer's disease (see below), an $\mathrm{MMSE}^{17}$ score $>10$, and community residence. Over a 1 year period, 492 persons seen at the Rush Alzheimer's Disease Center in Chicago met these criteria; 410 (83\%) agreed to participate. The study was approved by the Human Inves- 
tigations Committee of Rush-Presbyterian-St Luke's Medical Center.

At baseline, the mean age was 75.5 (SD 7.3); range: 45-95); mean education was 12.0 (SD 3.4) years (range: $3-24) ; 274(66.8 \%)$ were female; $61(14.9 \%)$ were African-American, and $349(85.1 \%)$ were white. The mean MMSE was 18.7 (SD 4.3); range: 11-29).

\section{CLINICAL EVALUATION}

At baseline, persons had a structured, uniform evaluation, which was repeated annually for 4 years, with examiners blinded to previously collected data. The procedures of the Consortium to Establish a Registry for Alzheimer's Disease (CERAD) ${ }^{21}$ were incorporated in the evaluation, which included a medical history, neurological examination, cognitive function testing, and informant interview; laboratory tests and brain scans were done at baseline but not at follow up unless clinically indicated. At each evaluation, prescription and over the counter medications were inspected, identified, and coded using the Medi-Span Data Base system. ${ }^{22}$

A board certified neurologist classified people for Alzheimer's disease and other conditions affecting cognitive or physical function. The diagnosis of Alzheimer's disease required a history of cognitive decline and evidence of impairment in memory and cognitive function. The criteria for probable Alzheimer's disease set forth by the joint working group of the National Institute of Neurological and Communicative Disorders and Stroke and the Alzheimer's Disease and Related Disorders Association (NINCDS/ADRDA) ${ }^{23}$ were met by 380 persons; 30 met NINCDS/ADRDA criteria for possible Alzheimer's disease because of the presence of another condition thought to contribute to cognitive impairment.

The neurological examination included administration of the entire motor portion of the unified Parkinson's disease rating scale (UPDRS). ${ }^{24} \mathrm{~A}$ previously established global measure of parkinsonism was derived from this scale and used in all analyses. At baseline, the mean score on this measure was 9.9 (SD 9.9); range: 0 to 51). Further information on the composition and metric properties of this measure is published elsewhere. ${ }^{25} 26$

\section{ASSESSMENT OF COGNITIVE FUNCTION}

Eighteen cognitive tests were administered at each evaluation and used to form six outcome measures. The primary measure was a composite based on 17 tests, excluding the MMSE. Test scores were converted to $\mathrm{z}$ scores, using the baseline mean and SD, and averaged to yield the composite measure. Because the MMSE has been used in many previous studies, it served as a secondary measure of global cognitive function. It has been suggested that psychotic symptoms may be differentially associated with specific facets of cognitive function. ${ }^{1027}$ To address this issue, the cognitive measures were subdivided into four domains, based on a previous factor analysis. Measures of memory, visuoconstruction, repetition, and naming (based on six, three, four, and four individual tests, respectively) were formed in the same way as the composite score, by converting raw scores to $\mathrm{z}$ scores and computing the average. Further psychometric information on the individual tests and factor analysis is provided elsewhere. ${ }^{20}$

ASSESSMENT OF HALLUCINATIONS AND DELUSIONS

At the baseline evaluation, the person with the greatest amount of daily contact with the participant was identified. Interview of this person took place at each evaluation, unless this person was unavailable in which case a secondary informant was used. Interviews were highly structured and were conducted by trained research assistants.

Delusions were assessed with seven questions which covered DSM-III- $\mathrm{R}^{28}$ subtypes of persecutory, grandiose, somatic, and jealous delusions. Misidentification syndromes were also included. Hallucinations were assessed with four questions which addressed auditory and visual experiences, inferred from the patient's behaviour or verbalisations. Misinterpretations of environmental stimuli were not treated as hallucinations. In a previous study, ${ }^{29}$ composite measures of delusions and hallucinations based on these items had adequate internal consistency (coefficient $\alpha=0.82$ for delusions and 0.71 for hallucinations) and stability over a 1 month retest interval ( $r=0.76$ for delusions and 0.79 for hallucinations). In the present study, delusions or hallucinations were regarded as present at baseline if at least one item indicated occurrence since the onset of dementia; on follow up, questions referred to occurrences since the previous examination. Insight regarding hallucinations was not assessed.

FOLLOW UP PARTICIPATION

Over $90 \%$ of survivors participated in each follow up evaluation. There were 141 deaths during the study, with 23 occurring before the first follow up evaluation. Longitudinal analyses required at least two valid scores on a given outcome measure. Among those who survived to the first follow up, $91.5 \%$ met this criterion for the composite measure of cognitive function (mean=3.8). There was an average of 3.7 valid hallucination and delusions scores for each person in this group. Additional information on follow up participation is provided elsewhere. $^{20}$

\section{DATA ANALYSIS}

Random effects regression models were used to estimate individual patterns of change in cognitive function over the study period and to test whether psychotic signs and other covariates were related to initial level of or rate of change in cognitive function. ${ }^{30}$ The models assumed that each person's individual path of change followed the mean path except for random effects that caused the initial level to be higher or lower and the rate of change to be faster or slower. Both of these random effects were assumed to follow a bivariate normal distribution, and the observed measurements 
were assumed to differ from a person's true path only by independent, identically distributed errors at each time of observation. These two components of between person variation were used to estimate growth curves for each person, which were plotted.

The random effects analyses were carried out in SAS PROC MIXED. ${ }^{31}$ The mean estimate indicates the average change in the cognitive score associated with a one unit change in the predictor. The standard error (SE) is estimated from the overall likelihood equation, and allows for inferences about the reliability of effects. The basic assumptions of the models involve linearity, normality, independence of errors, and homoscedasticity of errors. Linearity assumptions were checked analytically by including quadratic or other non-linear terms in models and graphically by plotting residuals against the predictors. Normality, independence, and homoscedasticity were evaluated by plotting estimated random effects in a scatter plot, by plotting residuals from each time point, and by examining univariate and bivariate summary statistics. We concluded from these procedures that model assumptions were adequately met. A more detailed explanation of the application of these models is contained in a previous publication. ${ }^{20}$

Results

Hallucinations were noted in $41.0 \%$ of the cohort at baseline, $39.7 \%$ at the second evaluation, $43.4 \%$ at the third, $33.6 \%$ at the fourth, and $31.1 \%$ at the fifth. Delusions were present in $54.7 \%$ at baseline, $47.5 \%$ at the second evaluation, $45.7 \%$ at the third, $34.3 \%$ at the fourth, and $29.8 \%$ at the fifth. Over the full study period, hallucinations were present on at least one evaluation in $69.5 \%$ and delusions in $80.2 \%$

Random effects models were used to test whether hallucinations or delusions were related to baseline level of or rate of change in cognitive function. To capitalise on all available data, the primary analyses used the composite measure of cognitive function. The initial model had terms for study time (in years), hallucinations (at baseline), and the interaction of hallucinations with time. This and subsequent analyses also included adjustment for demographic (age, education, sex, race) and clinical (parkinsonism) variables that can affect cognitive function. In this model (table 1), the cognitive score declined an average of 0.47 standard units per year (95\% confidence interval -0.39 to -0.54$)$ in those without hallucinations. Hallucinations were associated with a lower cognitive score at baseline, by an average of 0.33 units, and with an average decline per year of 0.69 units, which is an increase of $47 \%$ compared with those without hallucinations.

Figure 1 shows the distribution of the slopes estimated from this model for each subgroup. Rates of cognitive decline are generally greater in those with hallucinations than in those without them, but much variability is evident in both subgroups.

The primary analysis was repeated using the MMSE because of its extensive use in prior
Table 1 Summary of random effects models examining the relations of hallucinations and delusions with baseline level of and rate of change in the composite measure of cognitive function. Terms for age, education, sex, race, parkinsonism, and their interactions with time were also included in analyses

\begin{tabular}{lll}
\hline Model terms & Mean estimate & $S E$ \\
\hline Time & $-0.47^{\star \star \star}$ & 0.04 \\
Hallucinations & $-0.33^{\star \star \star}$ & 0.07 \\
Hallucinations $\times$ time & $-0.22^{\star \star \star}$ & 0.05 \\
Time & $-0.51^{\star \star \star}$ & 0.04 \\
Delusions & $-0.18^{\star \star \star}$ & 0.07 \\
Delusions $\times$ time & -0.08 & 0.05 \\
\hline
\end{tabular}

The mean estimate is the average change in the cognitive score associated with a one unit change in the model term. $\star \star \star \mathrm{p}<0.001$

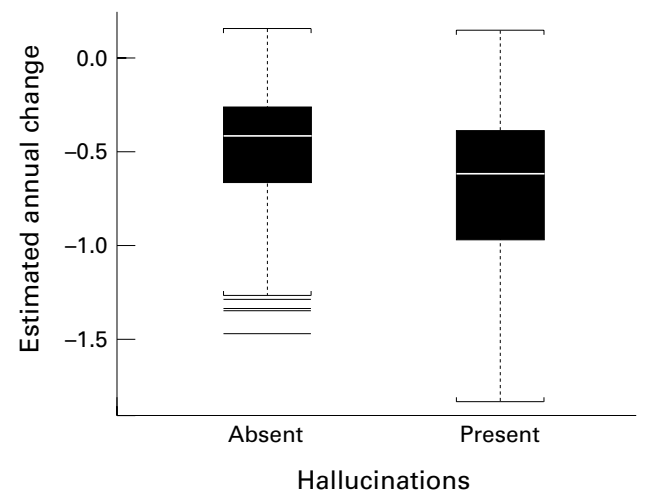

Figure 1 Distribution of estimated annual rate of change in composite measure of cognitive function in those with and without hallucinations at baseline. Estimates are from a random effects model adjusted for age, education, sex, race, and parkinsonism. Each box contains the middle 50\% of the distribution; the horizontal lines outside the brackets are outliers.

longitudinal studies. The MMSE score declined an average of 3.19 points per year (SE $0.16)$ in those without hallucinations. In those with hallucinations, MMSE score was 1.85 points (SE 0.51) lower at baseline and declined an average of 0.59 additional points per year (SE $0.23, \mathrm{p}<0.01$ ). This represents an $18 \%$ increase in the rate of MMSE decline associated with hallucinations.

Three potentially confounding factors were examined in subset analyses. Firstly, because antipsychotic medications can affect psychotic signs and cognition, the analysis was repeated excluding those on such medications at any evaluation point. Compared with the primary model, there was a slight reduction in the association of hallucinations with baseline cognitive function, but the association with cognitive decline was comparable. Secondly, because other conditions such as stroke can contribute to hallucinations and cognitive impairment, the analysis was repeated excluding those with these conditions, and comparable results were obtained. Thirdly, to see if mortality influenced the findings, the analysis was restricted to people who survived the study period. Results were not substantially changed.

To see if hallucinations were related to some forms of cognitive function but not to others, the primary analysis was repeated on the summary measures of memory, visuoconstruction, repetition, and naming. Hallucinations were associated with lower baseline scores and more 


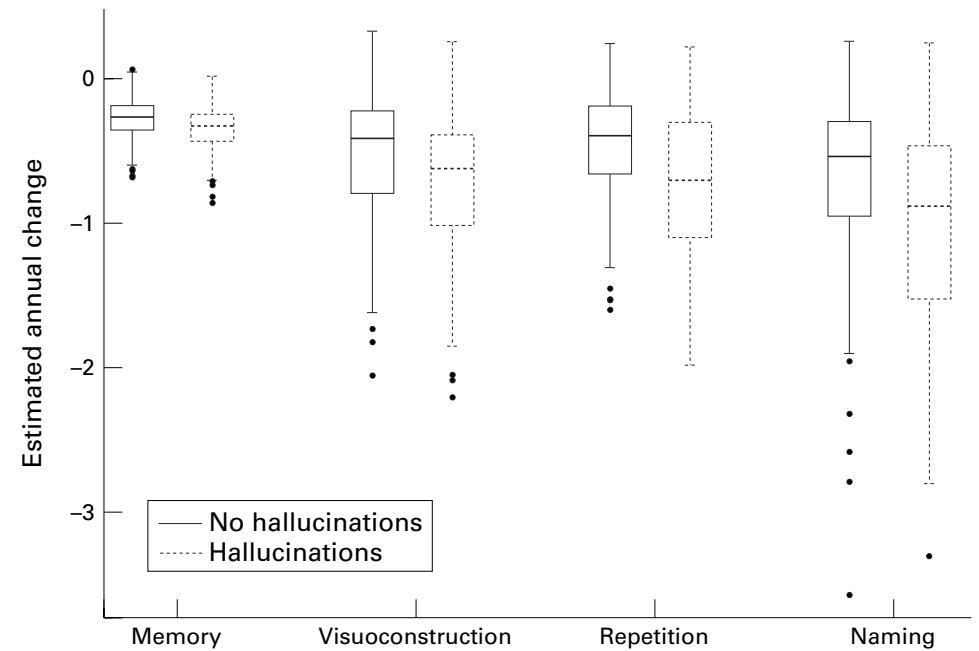

Figure 2 Distributions of estimated annual rate of change in summary measures of memory, visuoconstruction, repetition, and naming in those with and without hallucinations at baseline. Estimates are from random effects models adjusted for age, education, sex, race, and parkinsonism. Each box contains the middle 50\% of the distribution; the dots are outliers.

Table 2 Summary of random effects model examining the relation of subtypes of hallucinations with baseline level of and rate of change in the composite measure of cognitive function. Terms for age, education, sex, race, parkinsonism, and their interactions with time were also included in the analysis

\begin{tabular}{lll}
\hline Model terms & Mean estimate & $S E$ \\
\hline Time & $-0.48^{\star \star \star}$ & 0.04 \\
Visual hallucinations & $-0.20^{\star}$ & 0.10 \\
Visual hallucinations $\times$ time & -0.12 & 0.07 \\
Auditory hallucinations & -0.18 & 0.12 \\
Auditory hallucinations $\times$ time & -0.16 & 0.10 \\
Both hallucinations & $-0.50^{\star \star \star}$ & 0.09 \\
Both hallucinations $\times$ time & $-0.33^{\star \star \star}$ & 0.07
\end{tabular}

The mean estimate is the average change in the cognitive score associated with a one unit change in the model term. ${ }^{\star} \mathrm{p}<0.05 ;{ }^{\star \star \star} \mathrm{p}<0.001$

rapid decline on each measure. These results are graphically displayed in figure 2 . In those with hallucinations, compared with those without them, the average annual rate of decline was increased about $31 \%$ for memory $(\mathrm{p}<0.01), \quad 31 \%$ for visuoconstruction $(p<0.05), 48 \%$ for repetition $(p<0.001)$, and $44 \%$ for naming $(\mathrm{p}<0.001)$.

At baseline, hallucinations were exclusively auditory in $7.9 \%$ of the cohort, exclusively visual in $15.2 \%$, and both auditory and visual in $17.9 \%$. To see if cognitive decline differed in these subgroups, a model was run with terms for visual hallucinations, auditory hallucinations, both visual and auditory hallucinations, and their interactions with time (table 2). Rate of cognitive decline was substantially increased in those with both visual and auditory hallucinations. Rate of cognitive decline in those with only visual or auditory hallucinations was increased but not significantly so $(0.05<p<0.10$ for both interaction terms).

Finally, the analysis was repeated with a hallucination measure based on the full study period, rather than the initial evaluation alone, because this approach has been used in some prior studies. ${ }^{34615}$ In those who never hallucinated, cognitive score declined an average of 0.39 units annually (SE 0.05). Those who ever hallucinated had lower baseline cognitive func- tion, by an average of 0.23 units (SE 0.07), and declined an average of 0.61 units annually (SE 0.05), an increase of $93 \%$ compared with those who never hallucinated.

The association of delusional thinking and cognitive function was assessed in a similar series of analyses. The primary model (table 1) indicated that persons with delusions at baseline had a lower composite cognitive score at baseline than those without delusions. However, delusions were not related to rate of cognitive decline, as shown by the interaction term in the model. A series of secondary analyses, similar to those conducted for hallucinations, examined potentially confounding variables, different cognitive function measures, and a measure of delusions based on the full study period. Delusions were not significantly related to rate of cognitive decline in any analysis.

\section{Discussion}

This study examined the occurrence of hallucinations and delusions over a 4 year period and their relation to cognitive decline in a large cohort of people with Alzheimer's disease. Each sign was common. Hallucinations were present in $31 \%$ to $41 \%$ of persons at each evaluation and delusions in $30 \%$ to $55 \%$. The frequency of these signs ${ }^{32}$ and their relative stability over time ${ }^{33}$ are consistent with previous research.

The presence of either hallucinations or delusions at the baseline evaluation was associated with lower baseline level of cognitive function. In analyses that controlled for baseline level of cognitive function, demographic variables, and selected clinical factors including antipsychotic medications and parkinsonism, hallucinations were associated with more rapid decline on all cognitive measures; the increase in the average annual rate of decline on a composite measure of cognitive function was nearly $50 \%$. This association was mainly due to those who exhibited both visual and auditory hallucinations. By contrast, delusional thinking was not significantly related to cognitive decline.

There has been some previous evidence that hallucinations and delusions are differentially related to cognitive decline in Alzheimer's disease. Burns et $a l^{212}$ reported such a dissociation. However, their observation period was only 1 year and most patients had severe dementia at baseline. The dissociation was also reported by Chui et $a l,{ }^{8}$ but their findings were mixed and neuroleptic use was not adequately controlled. By contrast, three studies did not find a dissociation between hallucinations and delusions. ${ }^{91415}$ Each of these negative studies had sample sizes under 100, however, reducing statistical power and the ability to control for potentially confounding factors.

As noted above, the relation of psychotic signs to cognitive decline in Alzheimer's disease has been inconsistent in prior studies. Our results suggest several factors that may have contributed to this variability. Firstly, many prior studies have analyzed hallucinations and delusions together. ${ }^{3-7} 101113$ In view of the dissociation seen in the present study, this approach has probably weakened findings, 
especially because delusions are usually more common than hallucinations. Secondly, we classified the presence/absence of psychotic signs on the basis of the baseline alone or the full study period. Results for hallucinations were stronger with the second approach, and it is noteworthy that most previous studies using this approach have yielded positive results ${ }^{346}$ or trends. ${ }^{15}$ This may be because hallucinations occur sporadically in Alzheimer's disease and repeated evaluations over time reduce the likelihood of missing their occurrence. Thirdly, most previous longitudinal studies have used the MMSE. We found a substantially stronger effect for hallucinations with a composite measure of cognitive function than with the MMSE. This is probably because the composite measure is based on 17 individual tests of varying difficulty, thereby reducing floor and ceiling effects and other sources of measurement error which complicate assessment of change in cognitive function in Alzheimer's disease. ${ }^{34}$

Several factors increase confidence in the findings. Firstly, the clinical diagnosis of Alzheimer's disease was based on uniform application of widely accepted criteria and confirmed in a high proportion of those who have come to postmortem. ${ }^{20}$ Secondly, because of the large sample size, it was possible to control for potentially confounding demographic and clinical variables. Thirdly, findings were consistent across a range of global and specific cognitive function measures. Fourthly, the longitudinal study design, high rate of follow up participation with an average of three to four evenly spaced observations per person, psychometrically sound cognitive function measures, and random effects regression models permitted characterisation of individual paths of cognitive change, how each sign influenced these paths, and whether these influences were on initial level, rate of change, or both.

The study also has important limitations. Firstly, assessment of hallucinations and delusions is difficult in Alzheimer's disease. It is likely, however, that such problems were minimised in the present study by use of informant based measures of each sign that have been shown to yield high rates of agreement across interviewers and brief temporal intervals ${ }^{29}$ and by the efficiency of the longitudinal design in identifying sporadically occurring events. Secondly, because participants were selected from a specialised diagnostic and treatment centre, they are unlikely to represent the full range of Alzheimer's disease in the population. Research on these issues in population based samples is needed.

Progressive cognitive decline is the principal clinical manifestation of Alzheimer's disease. The rate at which people decline is highly variable, however, and difficult to predict. Our results suggest that knowledge of whether or not auditory or visual hallucinations have occurred can substantially improve prediction of subsequent course, even after other prognostic factors have been considered. For this reason, obtaining informant report about hallucinations is an important part of the clinical evaluation of Alzheimer's disease.

We thank Cheryl Bibbs and Vanessa Alston for coordinating the study, Todd Beck, for statistical programming, Judy McElhany for preparing the manuscript, and the Rush Alzheimer's Disease Center patients and staff for accommodating the study. The study was supported by National Institute on Aging grants RO1 AG09966 and AG10161.

1 Chen J-Y, Stern Y, Sano M, et al. Cumulative risks of developing extrapyramidal signs, psychosis, or myoclonus in the course of Alzheimer's disease. Arch Neurol 1991;48:11413.

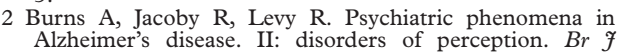
Psychiatry 1990;157:76-81.

3 Drevets W, Rubin E. Psychotic symptoms and the longitudinal course of senile dementia of the Alzheimer's type. Biol Psychiatry 1989;25:39-48.

4 Levy M, Cummings J, Fairbanks L, et al. Longitudinal assessment of symptoms of depression, agitation, and psychosis in 181 patients with Alzheimer's disease. Am f Psychosis in 181 patients with
chiatry 1996;153:1438-43.

5 Lopez O, Brenner R, Becker J, et al. EEG spectral abnormalities and psychosis as predictors of cognitive and functional decline in probable Alzheimer's disease. Neurology 1997;48:1521-5.

6 Rosen J, Zubenko G. Emergence of psychosis and depression in the longitudinal evaluation of Alzheimer's disease. Biol Psychiatry 1991;29:224-32.

7 Stern Y, Mayeux R, Sano M, et al. Predictors of disease course in patients with probable Alzheimer's disease. Neurology 1987;37:1649-53.

8 Chui H, Lyness S, Sobel E, et al. Extrapyramidal signs and psychiatric symptoms predict faster cognitive decline in Alzheimer's disease. Arch Neurol 1994;51:676-81.

9 Haupt M, Romero B, Kurz A. Delusions and hallucinations in Alzheimer's disease: results from a 2 year longitudinal study. International fournal of Geriatric Psychiatry 1996;11: 965-72.

10 Lopez O, Becker J, Brenner R, et al. Alzheimer's disease with delusions and hallucinations: neuropsychological and elecdelusions and hallucinations: neuropsychological and elec-
troencephalographic correlates. Neurology 1991;41:90612 .

11 Stern Y, Albert M, Brandt J, et al. Utility of extrapyramidal signs and psychosis as predictors of cognitive and functional decline, nursing home admission, and death in Alzheimer's disease: prospective analyses from the predictors study. Neurology 1994;44:2300-7.

12 Burns A, Jacoby R, Levy R. Psychiatric phenomena in Alzheimer's disease. I: Disorders of thought content. Br $\mathcal{F}$ Psychiatry 1990;157:72-6.

13 Lopez O, Wisniewski S, Becker J, et al. Psychiatric medication and abnormal behavior as predictors of progression in probable Alzheimer's disease. Arch Neurol 1999;56:1266-72.

14 Miller T, Tinklenberg J, Brooks J, et al. Selected psychiatric symptoms associated with rate of cognitive decline in patients with Alzheimer's disease. F Geriatr Psychiatry Neurol 1993;6:235-8.

15 Mortimer J, Ebbitt B, Jun S-P, et al. Predictors of cognitive and functional progression in patients with probable and functional progression in patients with

16 Teri L, McCurry S, Edland S, et al. Cognitive decline in Alzheimer's disease: a longitudinal investigation of risk factors for accelerated decline. $\mathcal{F}$ Gerontol A Biol Sci Med Sci 1995;50A:M49-55

17 Folstein M, Folstein S, McHugh P. Mini-mental state: a practical method for grading the mental state of patients for the clinician. F Psychiatr Res 1975;12:189-98.

18 Galasko D, Corey-Bloom J, Thal L. Monitoring progression in Alzheimer's disease. F Am Geriatr Soc 1991;39:932-41.

19 Galasko D, Abramson I, Corey-Bloom J, et al. Repeated exposure to the mini-mental state examination and the information-memory-concentration test results in a practice effect in Alzheimer's disease. Neurology 1993;43:155963.

20 Wilson R, Gilley D, Bennett D, et al. Person-specific paths of cognitive decline in Alzheimer's disease and their relation to age. Psychol Aging 2000;15:18-28.

21 Morris J, Drazner M, Fulling K, et al. Clinical and Morris J, Drazner $M$, Fulling $\mathrm{K}$, et al. Clinical and
pathological aspects of parkinsonism in Alzheimer's pathological aspects of parkinson
disease. Arch Neurol 1989;46:651-7.

22 Medi-Span. Master drug data base documentation manual. Indianapolis: Medi-Span, 1995

23 McKhann G, Drachman D, Folstein M, et al. Clinical diagnosis of Alzheimer's disease: report of the NINCDSADRDA Work Group under the auspices of Department of Health and Human Services Task Force on Alzheimer's Disease. Neurology 1984;34:939-44.

24 Fahn S, Elton R. Unified Parkinson's disease rating scale. In: Fahn S, Marsden C, Goldstein M, et al, eds. Recent developments in Parkinson's disease. Vol 12. Florham Park, NJ: MacMillan Healthcare Information, 1987:153-63.

25 Bennett D, Shannon K, Beckett L, et al. Metric properties of nurses' ratings of parkinsonian signs with a modified nurses ratings of parkinsonian signs with a modified
unified Parkinson's disease rating scale. Neurology 1997;49: 1580-6.

26 Bennett D, Shannon K, Beckett L, et al. Dimensionality of parkinsonian signs in aging and Alzheimer's disease. 7 Gerontol A Biol Sci Med Sci 1999;54A:M191-6. 
27 Jeste D, Wragg R, Salmon D, et al. Cognitive deficits of patients with Alzheimer's disease with and without
delusions. Am f Psychiatry 1992;149:184-9.

28 American Psychiatric Association. Diagnostic and statistical manual of mental disorders, 3rd ed, revised. Washington, DC: American Psychiatric Association, 1987.

29 Gilley D, Wilson R, Beckett L, et al. Psychotic symptoms and physically aggressive behavior in Alzheimer's disease. $f$ Am Geriatr Soc 1997;45:1074-9.

30 Laird N, Ware J. Random-effects models for longitudinal data. Biometrics 1982;83:963-74.
31 SAS Institute. SAS/STAT software: changes and enhancements through release (version 6.12). Cary, NC: SAS Institute, 1997.

32 Wragg R, Jeste D. Overview of depression and psychosis in Alzheimer's disease. Am f Psychiatry 1989;146:577-87.

33 Devanand D, Jacobs D, Tang M-X, et al. The course of psychopathologic features in mild to moderate Alzheimer's disease. Arch Gen Psychiatry 1997;54:257-63.

34 Kukull W. Problems in measuring and interpreting cognitive decline. f Am Geriatr Soc 1998;46:1578-9. 